\title{
EFFECTS OF HOLDING TEMPERATURE ON BAINITE TRANSFORMATION IN CR-MO STEEL
}

\author{
M. Sarizam ${ }^{1 *}$ and Y. Komizo ${ }^{2}$ \\ ${ }^{1 *}$ Faculty of Earth Science, University Malaysia Kelantan \\ Jeli Campus, Locked Bag No.100, 17600 Jeli, Kelantan, Malaysia \\ *Email: sarizam@umk.edu.my \\ Phone: +609-9477264; Fax: +609-9477032 \\ ${ }^{2}$ Joining and Welding Research Institute, Osaka University \\ 11-1 Mihogaoka, Ibaraki City, Osaka, Japan, 567-0047
}

\begin{abstract}
The effect of holding temperature on the variant selection mechanism during bainite transformation was investigated in $2 \mathrm{Cr}-1 \mathrm{Mo}$ steel. The transformation behaviour was studied by using high temperature laser scanning confocal microscopy (LSCM) via in situ observation. The specimen was heat treated to $1350{ }^{\circ} \mathrm{C}$ and was held for $60 \mathrm{~s}$ before being cooled to $560{ }^{\circ} \mathrm{C}$ and $580^{\circ} \mathrm{C}$. In both conditions, each specimen was isothermally transformed for 1 hour and cooled to room temperature. The heat-treated specimen was then analysed using the electron backscattered diffraction (EBSD) method. As a result, variant preferential selection is more prominent at a higher holding temperature, which coarsened the block and packet boundary and is connected with a low misorientation angle variant.
\end{abstract}

Keywords: Variant analysis; bainite; phase transformation; heat affected zone (HAZ); EBSD

\section{INTRODUCTION}

Bainite has become more important in steel technology nowadays due to the hope of achieving the best balance between strength and ductility $[1,2]$. The crystallography of bainite is said to closely resemble the traits of lath martensite in terms of orientation and packet formation, which is the lath martensite in low carbon steel, a prior austenite grain is divided into packets, lath in the same habit plane with respect to its matrix, and each packet is divided into blocks [3]. It is reported that this three-level hierarchy in its morphology plays a crucial role in improving the toughness of bainitic steel $[4,5]$. To date, several researches have been done to analyse the transformation of upper bainite by means of the EBSD method. However, until now, in situ observation of the bainite transformation in a single prior austenite grain which is associated with variant analysis of crystallographic packets has scarcely been studied. In the present study, in situ observation of phase transformation is conducted by using LSCM to grasp the block formation sequences in a single prior austenite grain, and variant analysis will then be done in accordance with the information. The focus of variant analysis here is on the orientation relationships of each block formed at different holding temperatures. Thus, the effect of the temperature on the variant selection mechanism can be identified. 


\section{EXPERIMENTAL METHODS}

\section{Experimental Set-Up}

Cr-Mo steel $(\mathrm{Fe}-0.14 \% \mathrm{C}-2.14 \% \mathrm{Cr}-0.86 \% \mathrm{Mo}$ wt $\%)$ was used in the present study. By using high temperature LSCM, the specimen, $5 \mathrm{~mm}$ in diameter and $1 \mathrm{~mm}$ in thickness, was placed in an alumina crucible and held in a platinum holder inside the furnace. Under a controllable condition, the specimens were then austenized at $1350{ }^{\circ} \mathrm{C}$ for $60 \mathrm{~s}$. After that, each specimen was cooled to $560{ }^{\circ} \mathrm{C}$ and $580{ }^{\circ} \mathrm{C}$ at $-15^{\circ} \mathrm{C} / \mathrm{s}$ and was held at this temperature for $3600 \mathrm{~s}$ before being cooled at room temperature. The transformation phase during this process was displayed on a monitor and was simultaneously recorded along with the image of 30 frames/s. An elaborate description of LSCM was detailed in our previous research [6]. The heat-treated specimens were measured for the crystal orientation at room temperature and were analysed using SEM/EBSD. The accelerating voltage of SEM (JEOL JSM-6400) was set at $20 \mathrm{kV}$, the electron beam diameter was about $0.2 \mu \mathrm{m}$ and the step size was 0.8 . The analysis results were then compared in order to study the behaviour of bainite transformation.

\section{Analysis}

It is well known that bainitic ferrite, like lath martensite, nearly holds the Kurdjumov-Sachs (K-S) relationship with respect to its austenite matrix [3-5, 7-10]. Figure 1(a) shows an example of orientation measurements for the bainitic ferrite formed in a single austenite grain, while Figure 1(b) shows the ideal orientation distribution for all the 24 variants of the K-S orientation relationship [7]. Determination of the variant was done by comparing these two pole figures. Figure 2 shows the relationship between neighbouring variants in respect of the coincidence site lattice (CSL) for the 24 variants [5].

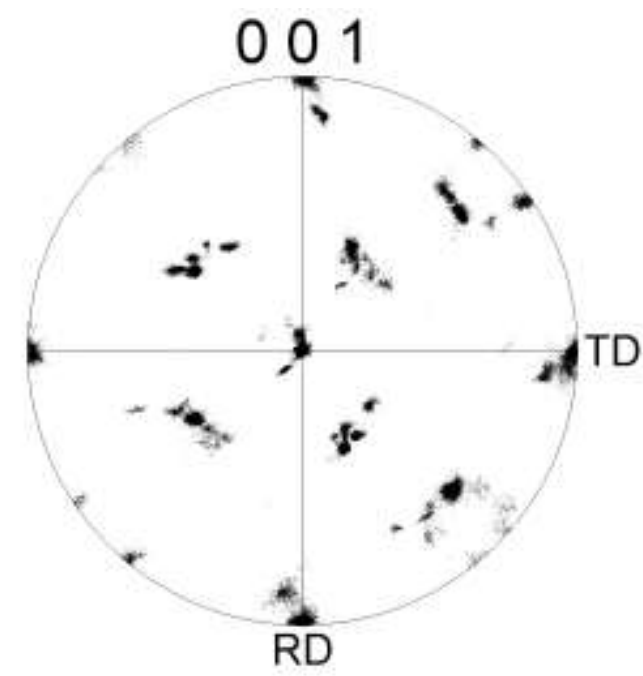

(a)

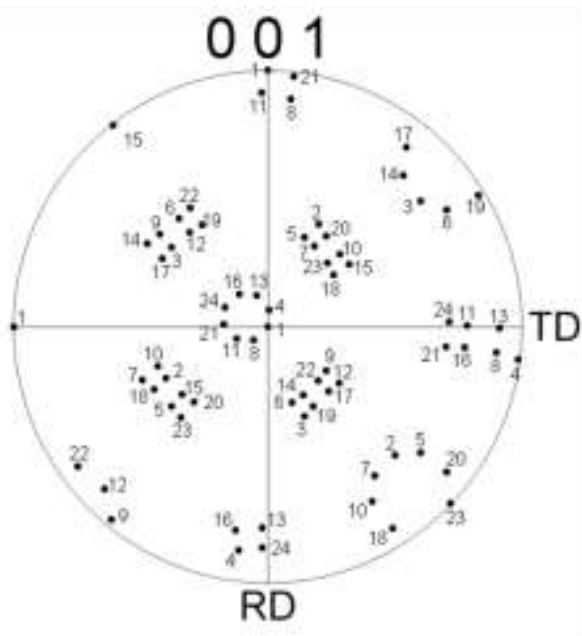

(b)

Figure 1. (a) A $001 \alpha$ pole figure of EBSD analysis data for bainitic ferrite held at $560^{\circ} \mathrm{C}$ for 1 hour, and (b) Configuration of $<001>\alpha$ poles for the K-S variants adjusted to (a). 


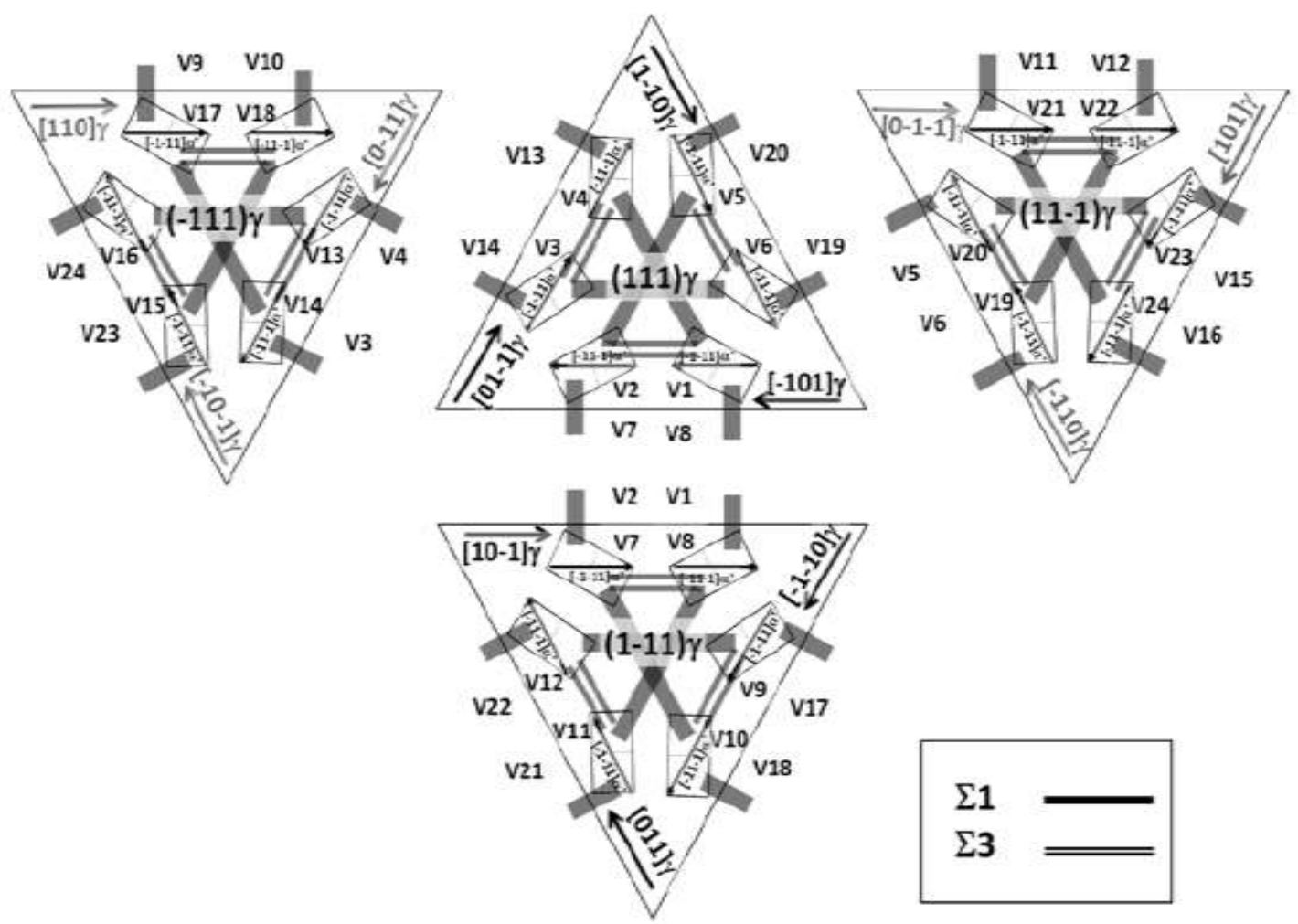

Figure 2. Illustration of the 24 crystallographic variants (V1-V24) evolving in the $\{111$ \}austenite plane in the K-S orientation relationship. The triangles and rectangles indicate the $\{111\}$ austenite (FCC) plane and (011) martensite (BCC) plane respectively [10].

The determination of CSL especially for $\Sigma 1$ and $\Sigma 3$ can be done easily by using this illustration. For example, V1 and V2, or V7 and V8, are recognized as a $\Sigma 3$ twinning relationship. Meanwhile, V1 and V8 or V2 and V7 are recognized as $\Sigma 1$. Moreover, it is understood that those variants which have a connection with V1 (V1-V4-V13-V16-V24-V21-V11-V8) are defined as belonging to the same Bain correspondence area. It is known that there are three different types of Bain correspondence area in one single austenite grain [10-12]. In this study, these Bain areas are subjected to blocks that exists in one packet. During the analysis, these three areas are defined in three different colours, called a BP map. In addition to this map, a close-packed (CP) map was used in order to identify different packets in the bainite microstructure (there are four packets in one single austenite grain, where one packet consists of six variants sharing a similar habit plane). In conjunction with this, variant analysis on bainite was done according to the pole figure method.

\section{RESULTS AND DISCUSSION}

\section{Isothermal Bainite Transformation Behaviour}

\section{Holding temperature at $560^{\circ} \mathrm{C}$}

Figure 3 shows the isothermal transformation behaviour observed at $560{ }^{\circ} \mathrm{C}$ holding temperature. Generally, the transformation from austenite to bainite starts after just $16 \mathrm{~s}$ 
of incubation period and reaches the saturated condition after $3567 \mathrm{~s}$.

The area marked in red shows the analysed area, while the numbered arrows describe the transformation sequences. The first bainitic ferrite block, Q1, as shown in Figure 3(b), transformed after $25 \mathrm{~s}$. It was nucleated from inside the grain and slowly grew in elongated directions until being stifled by the opposite grain boundary. $10 \mathrm{~s}$ after the first nucleation, a nucleation was observed at the same site but grew in the opposite direction and in a straight line compared to the previous one. Figure 3(c) shows the nucleation site for the next block formed, Q2, 49 s after incubation. Here, it is interesting to note that, with the exception of Q4, all of the bainitic ferrite blocks in this analysed grain nucleated from the same grain boundary. In the case of $\mathrm{Q} 4$, it is suggested that the transformation that occurred in a neighbouring grain becomes a factor for the nucleation in order to accommodate the strain involved during transformation. The transformation in the analysed grain ceased $138 \mathrm{~s}$ later.
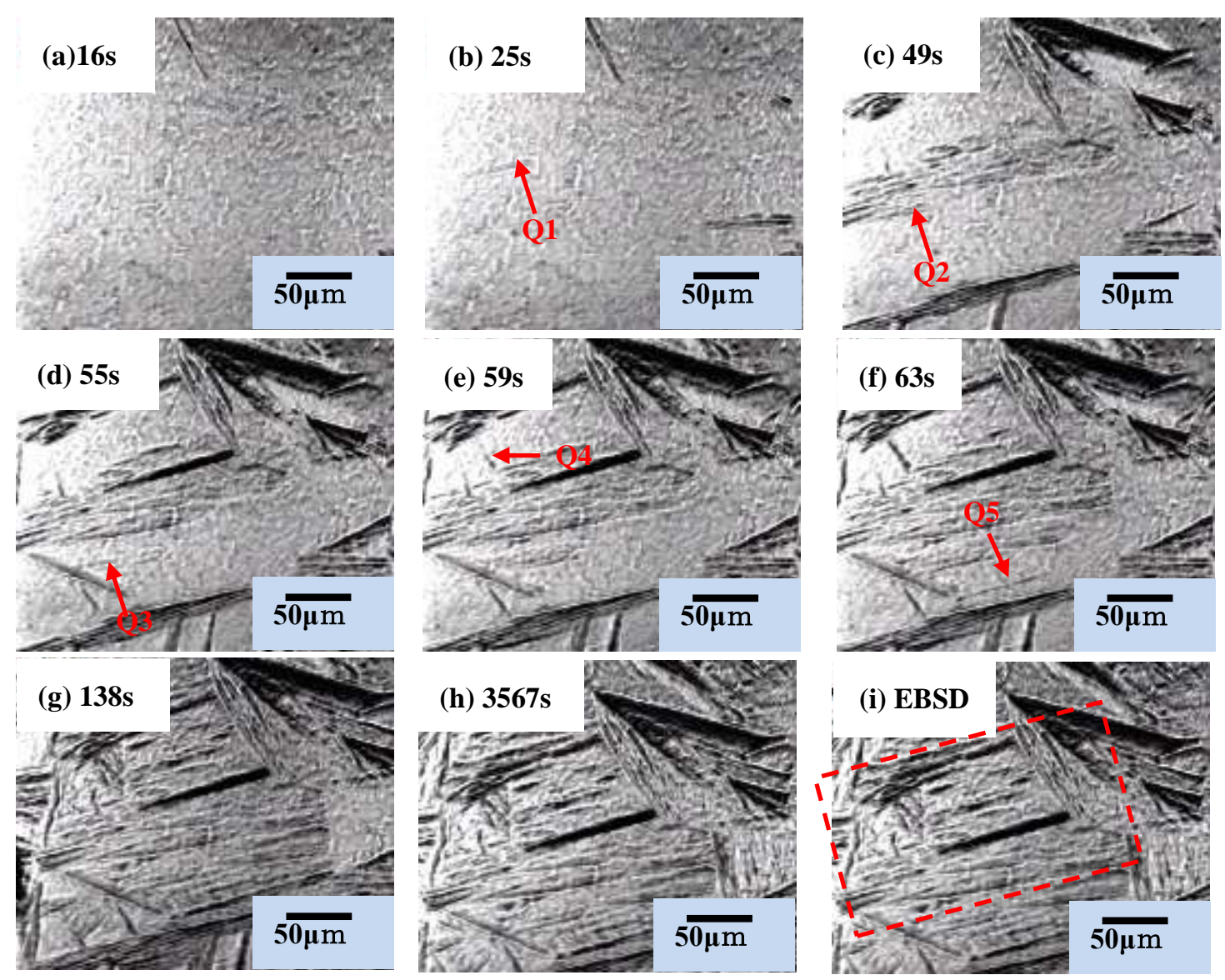

Figure 3. Isothermal transformation behaviour of Cr-Mo steel at $560{ }^{\circ} \mathrm{C}$ x 1 hour: (a) transformation started after $16 \mathrm{~s}$ incubation period, (b)-(f) nucleation and growth of bainitic ferrite lath are shown in sequences marked as Q1, Q2, Q3, Q4 and Q5 which had nucleated after the indicated incubation period, (g) transformation of the analysed grain ceased after $138 \mathrm{~s}$, (h) transformation of whole grain ceased after $3567 \mathrm{~s}$, (i) analysed grain. 


\section{Holding temperature at $580^{\circ} \mathrm{C}$}

Figure 4 shows the isothermal transformation behaviour observed at the $580{ }^{\circ} \mathrm{C}$ holding temperature. It is clear that the specimen exhibits very similar characteristics to when it was being held at $560{ }^{\circ} \mathrm{C}$. However, when it comes to the kinetics of transformation, the current holding temperature was observed to be slower and reached saturated conditions after a shorter incubation period. As shown in Figure 4(a) and 4(f), the transformation started after $5 \mathrm{~s}$ and ceased after $941 \mathrm{~s}$ of incubation. The red marked area in Figure 4 (g) shows the analysis area and the numbered arrows that appear in Figure 4(a)-(d) describe the transformation in sequences. The first block, T1, nucleated from the grain boundary after $17 \mathrm{~s}$ of incubation, then grew slowly in length and width simultaneously. As the transformation proceeded, the second and third blocks, T2 and T3, were observed to have nucleated at the same grain boundary after $25 \mathrm{~s}$ and $28 \mathrm{~s}$ respectively. The transformation was observed to cease after a $93 \mathrm{~s}$ incubation period.
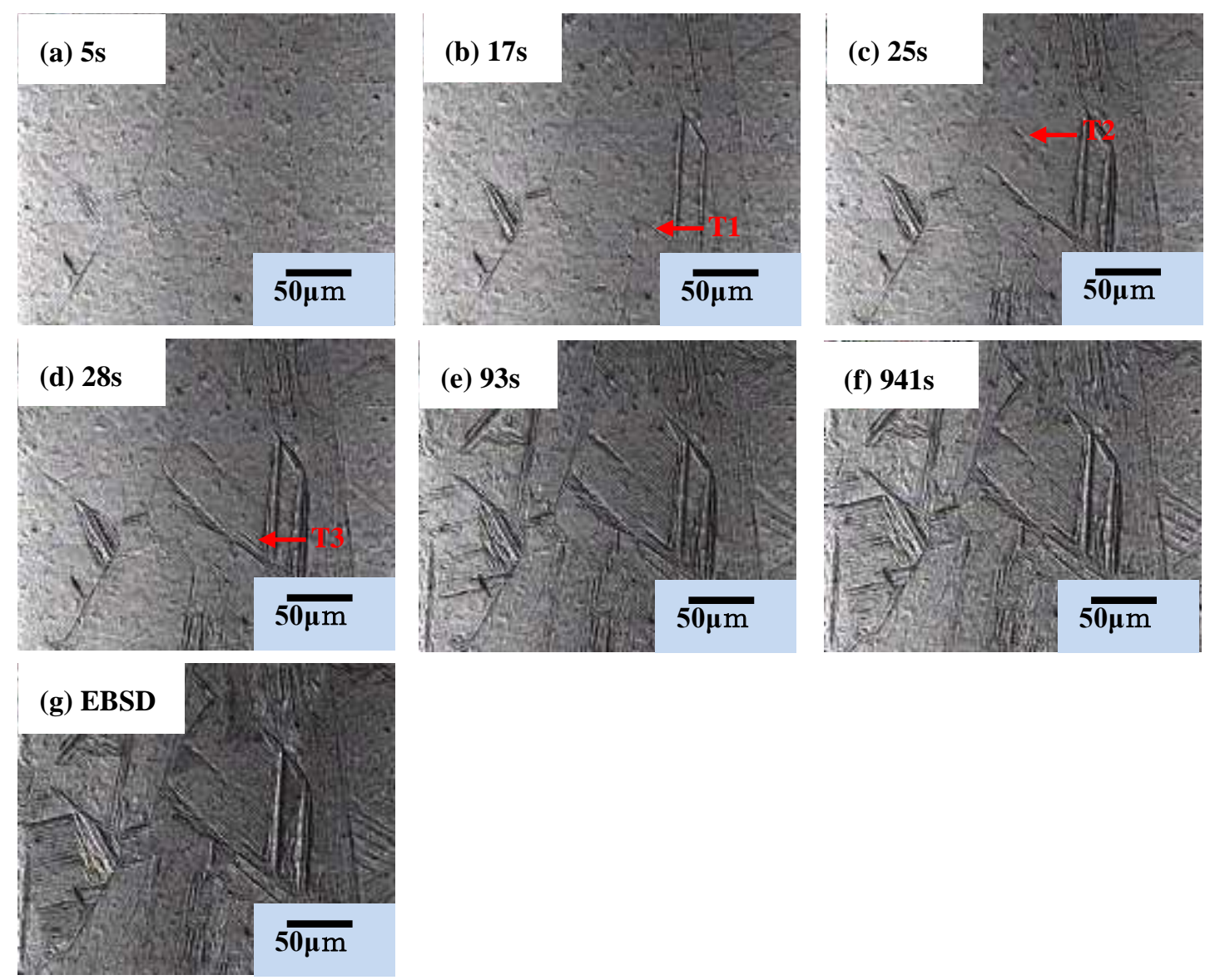

Figure 4. Isothermal transformation behaviour of Cr-Mo steel at $580{ }^{\circ} \mathrm{C}$ x 1 hour: (a) transformation started after $5 \mathrm{~s}$ of incubation period, (b)-(d) nucleation and growth of bainitic ferrite block shown in sequences marked as T1, T2 and T3 which had nucleated after the indicated incubation period, (e) transformation of analysed grain ceased after $93 \mathrm{~s}$, (f) transformation of whole grain ceased after $941 \mathrm{~s},(\mathrm{~g})$ analysed grain. 


\section{Variant Analysis}

In this study, the crystal orientation of the bainitic ferrite was measured by using EBSD and determination of the variants was done in the same area by using the pole figure method. Figure 5 shows the crystal orientation for specimens that had transformed under the isothermal conditions. Figure 5(a) and (c) are the inverse pole figure (IPF) of the bainitic ferrite, while Figure 5(b) and (d) describe the fraction numbers of misorientation angles in graph form subject to Figure 5(a) and (c) respectively. Different colours are used to distinguish variant differences. As shown in Figure 5(a) for the $560{ }^{\circ} \mathrm{C}$ holding temperature, V1 in red was seen to be the dominant variant. However, there was also some formation of V2 within the V1, which contributed about $47.9 \%$ of the high angle misorientation in the graph. It is known that the relationships between these two variants, V1 and V2, are CSL $\Sigma 3$. From the in situ observation by LSCM, it is clear that the transformation ceased after $3567 \mathrm{~s}$ of holding time, and the transformation was said to be an incomplete transformation [13] so the untransformed austenite remained in between the bainitic ferrite blocks. Thus, the formation of V2 between the V1 blocks is suggested to have occurred during the continuous cooling after 1 hour of holding time. However, there is no evidence regarding this matter in proving the differences that may exist in the microstructure crystallography of bainitic ferrite transformed isothermally and athermally, except for this visual observation during LSCM. In addition, the formation is observed of variant boundaries V1 and V3 with a high misorientation angle, recorded as $60^{\circ}$, and variants which have a CSL $\Sigma 11$ relationship such as V1 and V6, and V2 and V3, that also contribute to the fraction of high misorientation angle in the graph. Anyhow, the transformation of V1 was dominant in contributing the fraction of low misorientation angle shown in Figure 5(b). Moreover, specific variant pairs formed during the transformation, such as V1 and V4, V2 and V5 and V3 and V6. All of these pairs have a low misorientation angle of $10.5^{\circ}$. Thereafter, when the transformation temperature was increased to $580{ }^{\circ} \mathrm{C}$, the formation of $\mathrm{V} 1$ clearly dominated the grain. This phenomenon was probably caused by the tendency to form the specific variant and to finally coarsen the blocks. This result is in line with the result by Furuhara, Kawata [14], who suggested that coarser blocks were formed by increasing the transformation temperature.

\section{Morphology of Blocks and Packets}

The so-called BP map and CP map were used to clarify the relationships of the determined variants in Figure 6 with the corresponding Bain strain and related closed-packed plane [10]. As for the BP map, there are three Bain groups in the grain and these are illustrated in three different colours, red, green and blue, which refer to the three $<001>\gamma$ directions. Likewise, for the CP map, there are ideally four packets in the grain, subject to the parallel close-packed planes $\{011\} \alpha / /\{111\} \gamma$ in line with K-S orientation relationships and these four planes are illustrated in four different colours, red, green, blue and yellow.

Figure 6 illustrates the BP and CP maps. The BP map shows that there is no variant with a high misorientation angle within the same Bain correspondence area. This can be confirmed easily in the analysis, wherein there is no black or white line in the areas represented by red, green or blue colours that refers to $<001>\gamma$ directions. Here, the black line refers to a high misorientation angle and the white line refers to twinning relationships. However, from the result of $\mathrm{CP}$ map analysis, the formation of a high 
misorientation angle and twinning relationship variants in the same packet area can be observed clearly by looking at the black and white line. Similar to the BP map, the black line refers to a high misorientation angle and the white line refers to twinning relationships. Then, by comparing both maps, it can be concluded that the packet boundary is connected with low misorientation angle variants which belong to the same Bain group.

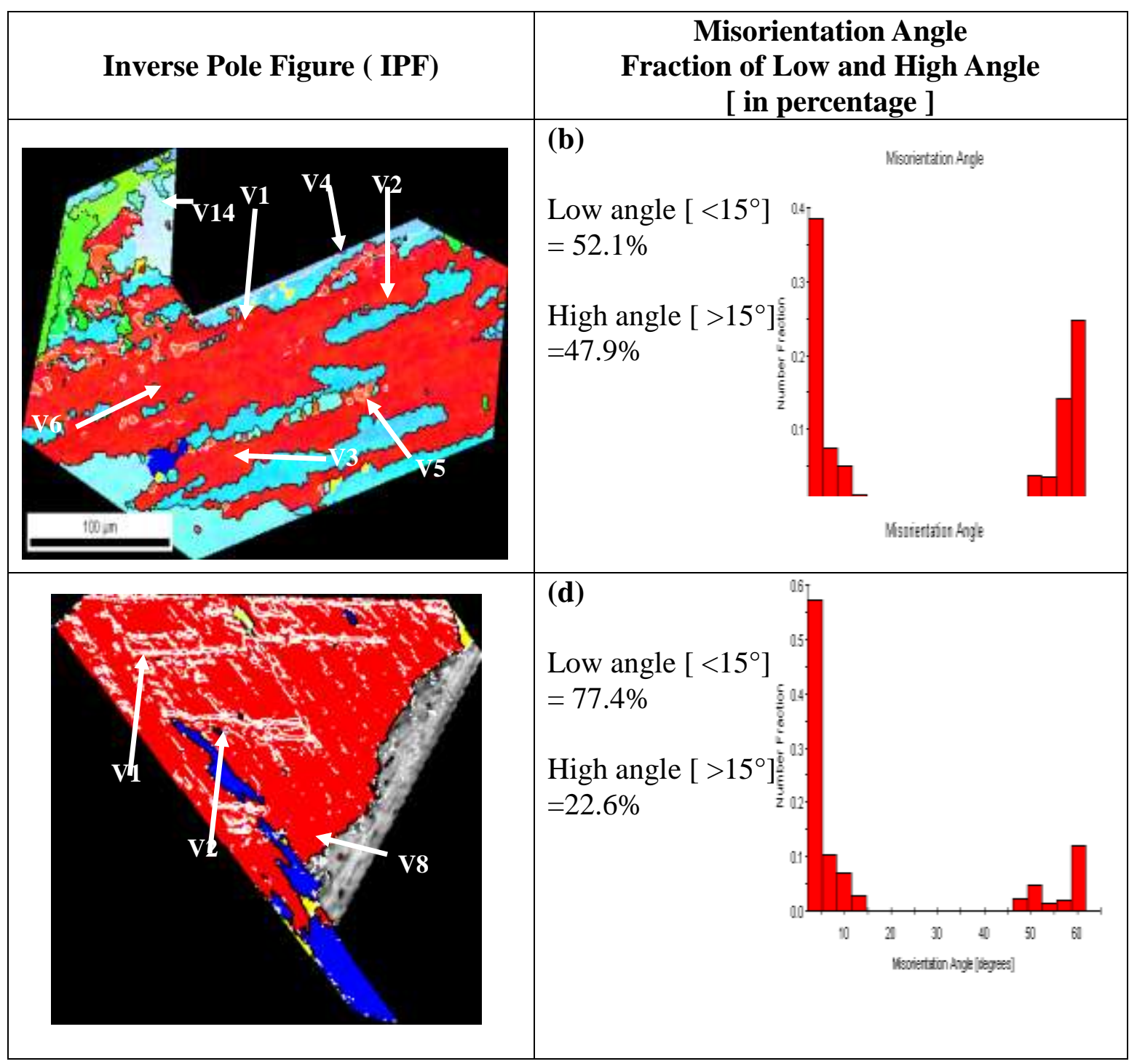

Figure 5. Variant determination and distribution of low angle and high angle misorientation in each condition: (a), (b) $560{ }^{\circ} \mathrm{C}$, (c), (d) $580{ }^{\circ} \mathrm{C}$.

\section{Correlation Between Block Formation Sequences and Variant}

Figure 7 shows the correlations between the transformation sequences and the formation of variants in the grain for the case of isothermal transformation at $560{ }^{\circ} \mathrm{C}$ holding temperature. By comparing the LSCM and EBSD analysis results, the first block, Q1, can be matched to V1, which is red in colour. Then, after some while, of the blocks that had transformed simultaneously, marked as Q2, one had nucleated from the same grain boundary of Q1, while the other one was classified as a secondary block since it had 
nucleated from the primary block, which is also identified as V1. The same is the case for Q3 and Q4, where both of them were identified as V1. This phenomenon might occur due to a preferential variant selection mechanism, where there is a tendency to form a specific variant which in turn causes the formation of a coarser block. This phenomenon can also be related to plastic deformation in the austenite phase in order to relax the strain during the formation of bainitic ferrite.

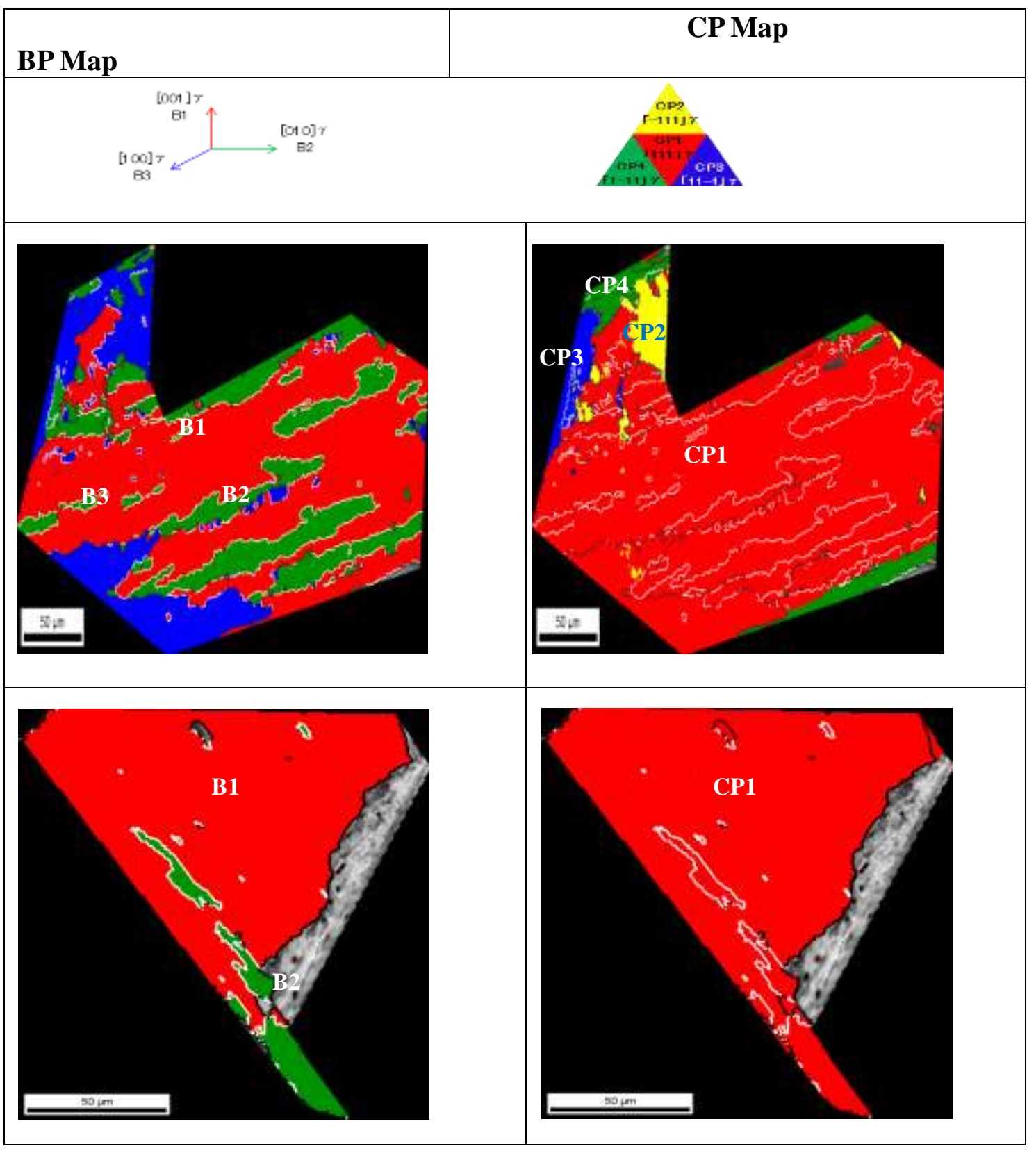

Figure 6. BP map and CP map for each condition: (a), (b) $560{ }^{\circ} \mathrm{C}$, (c), (d) $580{ }^{\circ} \mathrm{C}$.

Next, Figure 8 below shows the correlations between the transformation sequences and the formation of variants in the grain that had undergone an isothermal transformation at $580{ }^{\circ} \mathrm{C}$ holding temperature. The first block, T1, can be matched as $\mathrm{V} 2$ and caused a twinning to become V1 during the growth process. After that, the second block, T2, transformed and was followed by T3 after a while. T2 was recognized 
as V1 and T3 was V2, which causes twinning to become V1 during the growth process. Then, the growth process continues and develops in forming a coarse packet.
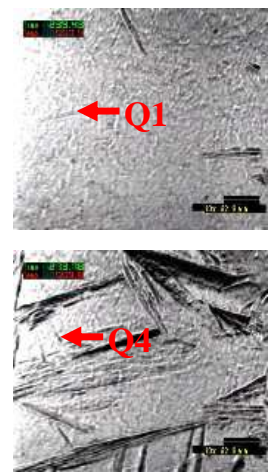
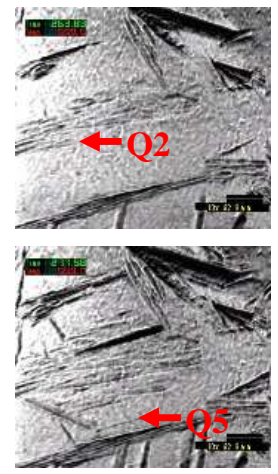
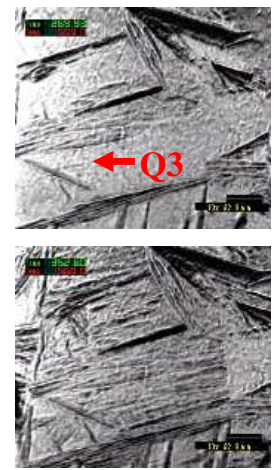

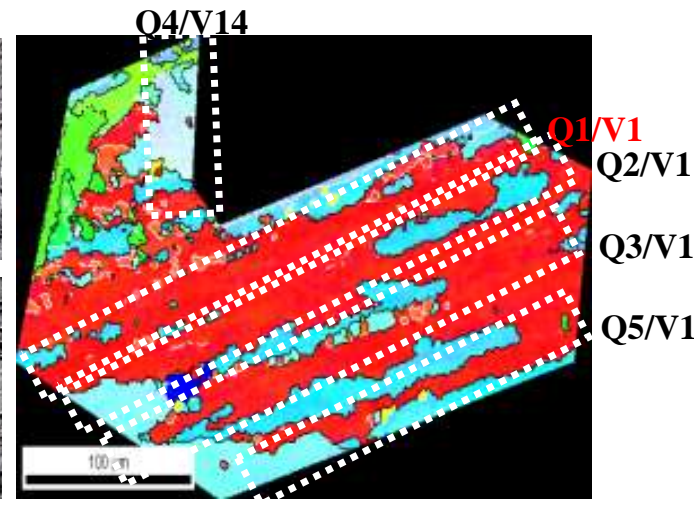

Figure 7. Correlations between transformation sequences by LSCM to be matched with variants formed in the grain for the case of $560{ }^{\circ} \mathrm{C}$ holding temperature
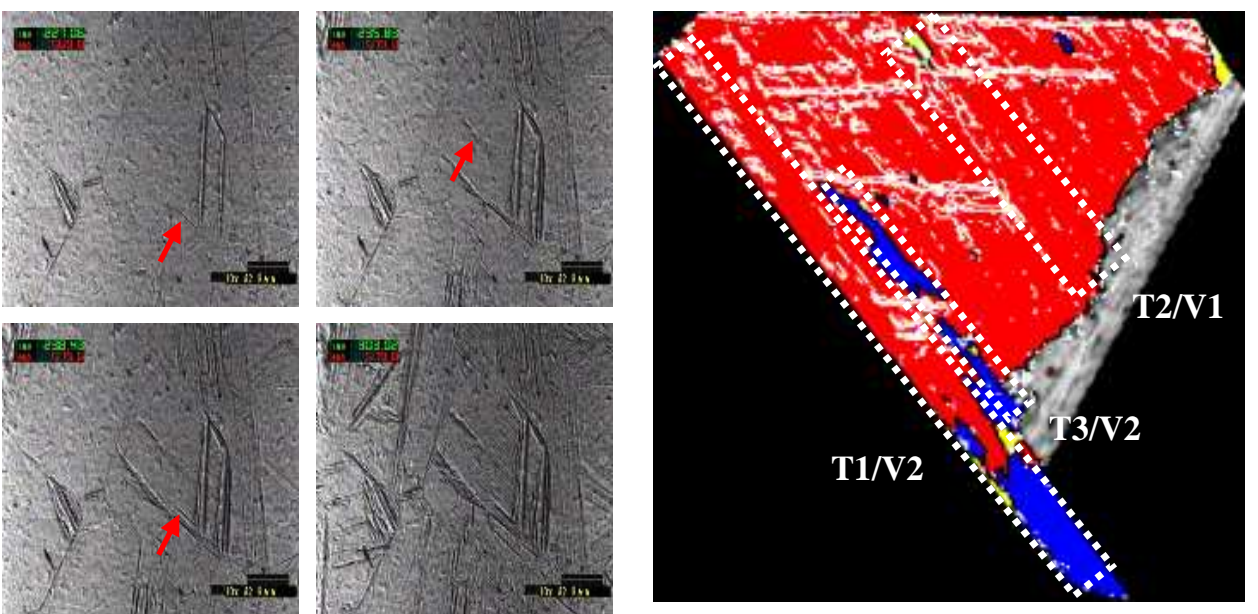

Figure 8. Correlations between transformation sequences by LSCM to be matched with variants formed in the grain for the case of $580{ }^{\circ} \mathrm{C}$ holding temperature.

\section{Effect of Holding Temperature}

Table 1 is a summary of data for the incubation times, the start and finish for every transformation temperature, and its relationship with an average block size. From the data, it can be concluded that the transformation temperature has a linear relationship with the average block size, where a higher transformation temperature resulted in a higher value of the average block size. This result is in line with the results reported by Furuhara, Kawata [14], suggesting that finer microstructure of bainitic ferrite can be obtained at a lower transformation temperature. Figure 9 shows the fraction of variants that have a CSL relationship, while the line graph shows the tendency to form a high misorientation angle at each of the mentioned temperatures. From these two results, it seems that there is a close relationship between the decreasing of the CSL $\Sigma 3 /$ high angle and the increasing average block size by increasing the transformation temperature. The formation of different textures at low and high temperature suggests that isothermal bainite transformation is driven by two mechanisms: the plastic-accommodation that 
normally occurs at high temperature, and self-accommodation at low temperature.

The variant analysis result has revealed that there is a tendency to form the same orientation of bainitic ferrite block (V1, which is represented in red in both cases) at both holding temperatures. Moreover, the formation of blocks in one observed packet is found to increase by decreasing the transformation temperature. This suggests that there is a variant selection in the nucleation of bainitic ferrite at the austenite grain boundary during isothermal transformation, and the effects can be seen more clearly at a higher transformation temperature. The cause of this formation of preferred variants is the reductions of boundary energy and strain energy produced during nucleation [14]. It is said that the transformation driving force is lower in the case of a high transformation temperature, and this will strengthen the preferential variant selection for nucleation of bainitic ferrite. The high temperature enables the untransformed austenite to deform plastically with ease in order to accommodate the transformation strain during development of the bainitic microstructure, which then coarsens the block.

Table 1. Effect of transformation temperature on the average block size.

\begin{tabular}{cccc}
\hline Transformation & \multicolumn{2}{c}{ Incubation Time [s] } & Average Block \\
\cline { 2 - 3 } Temperature $\left[{ }^{\circ} \mathrm{C}\right]$ & $\begin{array}{c}\text { Transformation } \\
\text { Started }\end{array}$ & $\begin{array}{c}\text { Transformation } \\
\text { Finished }\end{array}$ & \\
& 16 & 3567 & 4.04 \\
560 & 5 & 941 & 5.65 \\
\hline 580 & & & \\
\hline
\end{tabular}

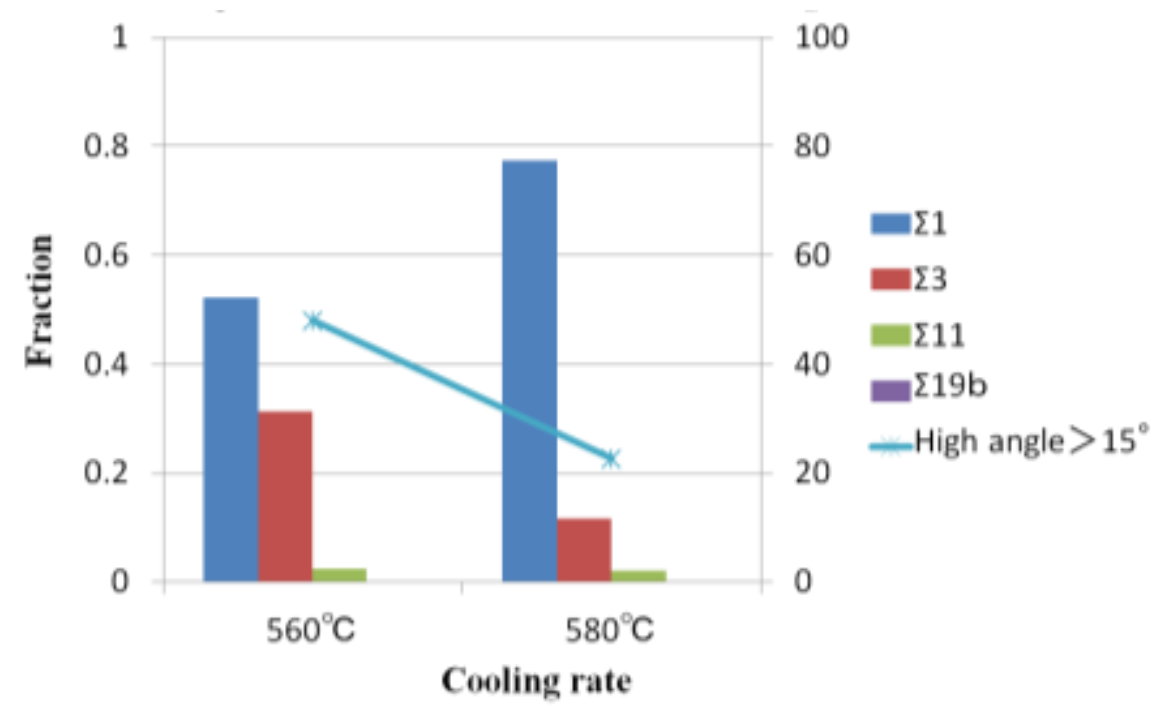

Figure 9. Graph showing the relationship between the fraction of variant formation and holding temperature.

The partition of carbon during transformation also has an impact on the variant selection mechanism. The holding temperature was in fact high, and for this reason, the carbon had enough time to partition into austenite and then became heterogeneous with carbon-enriched and carbon-depleted regions. It is reported that the variant selection is strong in heterogeneous nucleation, where the subsequent bainitic ferrite initiates from the carbon-enriched regions during the transformation development, and this criterion 
can be seen clearly during in situ observation by LSCM, especially for the case of the $580{ }^{\circ} \mathrm{C}$ holding temperature.

\section{CONCLUSIONS}

In this study, observation was concentrated on the transformation of bainite in a single austenite grain and the transformation sequence within the grain was analysed. By using the EBSD method, the orientation relationship of the transformed block was analysed. From the analysis, it can be concluded that:

i) The nucleation of bainitic ferrite block mainly initiates from the austenite grain boundary.

ii) The formation of same variants and specific variant pairs (V1 and V4, V2 and $\mathrm{V} 5, \mathrm{~V} 3$ and V6) proves the mechanism of variant preferential selection during bainite transformation. The mechanism caused the formation of coarser block, especially at the higher holding temperature.

iii) The packet boundary is connected with a low misorientation angle variant belonging to the same Bain group. The formation of CSL $\Sigma 1$ (belonging to the same Bain group) and CSL $\Sigma 11$ at the packet boundaries is an effect of strain accommodation during transformation.

\section{REFERENCES}

[1] Barbacki A. The role of bainite in shaping mechanical properties of steels. Materials Processing Technology 1995;53:57-63.

[2] Bhadeshia HKDH. Bainite in steels. London: Insitute of Materials Publication; 1992.

[3] Kawata H, Sakamoto K, Moritani T, Morito S, Furuhara T, Maki T. Crystallography of ausformed upper bainite structure in $\mathrm{Fe}-9 \mathrm{Ni}-\mathrm{C}$ alloys. Materials Science and Engineering: A. 2006;438-440:140-4.

[4] Furuhara T, kawata H, Moritani t, maki T. Crystallography of upper bainite in Fe-Ni-C alloys. materials Science and Engineering: A431. 2006:1279-88

[5] Furuhara T. Current opinion on definition of bainite structure in steels. Heat Treatment. 2012;50:22-7.

[6] Komizo Y, Terasaki H. Optical observation of real materials using laser scanning confocal microscopy Part 1. Science and Technology of Welding and Joining. 2011;16:56-60.

[7] Miyamoto G, Iwata N, Takayama N, Furuhara T. Variant selection of lath martensite and bainite transformation in low carbon steel by ausforming. Alloys and Compounds. 2012:1-5.

[8] Shen R, Cheng J, Wang K, Kang M. Nucleation of bainite around grain boundary. Material Science Technology. 1998;14:383-4.

[9] Luo CP. Crystallography of lath martensite and lower bainite in alloy steels. Material Science Engineering A 438-440. 2006:149-52.

[10] Zhang S, Morito,S.,Komizo.Y. Variant selection of low carbon high alloy steel in an austenite grain during martensite transformation. ISIJ International. 2012;52:510-5.

[11] Kitahara H, Ueji R, Tsuji N, Minamino Y. Crystallographic features of lath martensite in low-carbon steel. Acta Materialia. 2006;54:1279-88. 
[12] Kitahara H, Tsuji N. Crystallography of martensitic microstructure by means of FE-SEM/EBSP. Heat Treatment. 2010;50:186-93.

[13] Ohmori Y. Bainite transformation taken as the shear type transformation. Japan Metal Society Newsletter. Japan1990.

[14] Furuhara T, Kawata H, Morito S, Maki T. Crystallography of upper bainite in Fe-Ni-C alloys. Materials Science and Engineering. 2006. 